\title{
The Multistep Dissociation of Fluorine Molecules under Extreme Compression
}

Defang Duan ${ }^{1}$, Zhengtao Liu ${ }^{1}$, Ziyue Lin ${ }^{1}$, Hao Song ${ }^{1}$, Hui Xie ${ }^{1}$, Tian Cui ${ }^{2,1,}$, Chris J. Pickard $^{3,4}$, Maosheng Miao ${ }^{5,6, \dagger}$

${ }^{1}$ State Key Laboratory of Superhard Materials, college of Physics, Jilin University, Changchun 130012, China

${ }^{2}$ Institute of High Pressure Physics, School of Physical Science and Technology, Ningbo University, Ningbo, 315211, People's Republic of China

${ }^{3}$ Department of Materials Science \& Metallurgy, University of Cambridge, 27

Charles Babbage Road, Cambridge CB3 OFS, United Kingdom

${ }^{4}$ Advanced Institute for Materials Research, Tohoku University 2-1-1 Katahira, Aoba, Sendai, 980-8577, Japan

${ }^{5}$ Beijing Computational Science Research Center, Beijing 10084, P. R. China

${ }^{6}$ Department of Chemistry and Biochemistry, California State University Northridge, California 91220, United States 


\begin{abstract}
All elements that form diatomic molecules, such as $\mathrm{H}_{2}, \mathrm{~N}_{2}, \mathrm{O}_{2}, \mathrm{Cl}_{2}, \mathrm{Br}_{2}$, and $\mathrm{I}_{2}$ are destined to become atomic solids under sufficiently high pressure. However, as revealed by many experimental and theoretical studies, these elements show very different propensity and transition paths, due to the balance of reduced volume, lone pair electrons, and interatomic bonds. The study of $\mathrm{F}$ under pressure can illuminate this intricate behavior since $\mathrm{F}$, owing to its unique position on the periodic table, can be compared with $\mathrm{H}$, with $\mathrm{N}$ and $\mathrm{O}$, and also with other halogens. Nevertheless, $\mathrm{F}$ remains as the only element whose solid structure evolution under pressure has not been thoroughly studied. Using a large-scale crystal structure search method based on first principles calculations, we find that, before reaching an atomic phase, F solid transforms first into a structure consisting of $F_{2}$ molecules and $\mathrm{F}$ polymer chains and then a structure consisting of $\mathrm{F}$ polymer chains and $\mathrm{F}$ atoms, a distinctive evolution with pressure that has not been seen in any other elements. Both intermediate structures are found to be metallic and become superconducting, a result that add $\mathrm{F}$ to the elemental superconductors.
\end{abstract}


Many elements, such as $\mathrm{H}, \mathrm{N}, \mathrm{O}$, and $\mathrm{Cl}$, form diatomic molecules under ambient conditions. At high enough pressure they are destined to transform into atomic phases, accompanied by emerging properties such as metallization and high $T_{\mathrm{c}}$ superconductivity. Being the most abundant element, hydrogen, although containing only one electron in its shell, is very resistant to molecular dissociation and undergoes a series of structure changes before eventually transforming into atomic phases under extremely high pressures (> $550 \mathrm{GPa}$ ) [1]. Intermediate phases show complex structural features, such as the coexistence of two types of $\mathrm{H}$. Computational predictions [2,3] and the experimental evidence [4] reveal the corresponding phase contains alternating layers formed by two different types of $\mathrm{H}_{2}$ molecules.

The dissociation of elementary molecules and transformation into atomic phases is dictated by the number of valence electrons. More electrons delay the formation of atomic phases. $\mathrm{B}$ and $\mathrm{C}$ are stable as covalent solids under ambient pressures, whereas $\mathrm{N}$, due to its peculiar electron counting, forms the exceedingly strong $\mathrm{N} \equiv \mathrm{N}$ triple bonds and the most stable diatomic molecule. However, despite the presence of many metastable phases, its stable molecular phase evolves directly into an atomic "cubic gauche" (cg) phase at a modest pressure of about $110 \mathrm{GPa}[5,6]$. Its atomic phases show intricate structural variations, such as the predicted $\mathrm{N}_{10}$ diamondoid structure at $263 \mathrm{GPa}$ [7] and an all-nitrogen metallic salt at 2.5 TPa [8], respectively. As compared to $\mathrm{N}$, $\mathrm{O}$ has 6 valence electrons, a number that is suitable for a double $\mathrm{O}=\mathrm{O}$ bond and two lone electron pairs on each $\mathrm{O}$. Although $\mathrm{O}_{2}$ molecules consist of weaker bonds than $\mathrm{N}_{2}$, counterintuitively, they are much more resistant to dissociation and persist up to $1.9 \mathrm{TPa}$, beyond which they form polymeric spiral chain and zig-zag chain structures $[9,10]$. The next element is Fluorine, in which the diatomic bond is the weaker than for both $\mathrm{N}_{2}$ and $\mathrm{O}_{2}$, since the number of its valence electrons only permits the formation of single bonds. Nevertheless, no study, theoretical or experimental, has demonstrated the dissociation of $F_{2}$ under pressure. The x-ray powder diffraction studies of Meyer et al. indicated that $\alpha-\mathrm{F}_{2}$ is monoclinic with two candidate space groups $C 2 / m$ and $C 2 / c$ of solid fluorine at ambient pressure [11]. Lv 
et al performed $a b$ initio calculation and found that the $C 2 / m$ structure is dynamically unstable, and that the $C 2 / c$ structure transforms to $C m c a$ above $8 \mathrm{GPa}$ and remains stable up to $100 \mathrm{GPa}[12]$.

The behavior of $\mathrm{F}_{2}$ molecules under pressure can also be compared with diatomic molecules of other halogens such as $\mathrm{Cl}_{2}, \mathrm{Br}_{2}$ and $\mathrm{I}_{2}$. The metallization and dissociation of these molecules have been observed experimentally. Solid molecular $\mathrm{I}_{2}$ becomes mixed-molecular structure at $12.5 \mathrm{GPa}$ [13], an insulator to metal transition occurs at about $16 \mathrm{GPa}$ keeping molecular characteristic and dissociates into incommensurate modulated structure at about $23 \mathrm{GPa}$ [14]. The incommensurate structure transforms to an atomic phase with space group Immm at $30 \mathrm{GPa}$, then to $I 4 / m m m$ at $45 \mathrm{GPa}$, finally to $F m-3 m$ phase at $53 \mathrm{GPa}$ [15]. The $\mathrm{Cl}_{2}$ and $\mathrm{Br}_{2}$ solids share a common phase transition with $\mathrm{I}_{2}$, but the transition pressures are higher $[16,17]$. Recent experiments show that the mixed-molecular structure, metallization and molecular dissociation for $\mathrm{Cl}_{2}$ occurs at about 130, 200 and $258 \mathrm{GPa}$, respectively [18]. Remarkably, both $\mathrm{I}$ and $\mathrm{Br}$ are found to become superconducting with $T_{\mathrm{c}}=1.2 \mathrm{~K}$ and $T_{\mathrm{c}}=1.5 \mathrm{~K}$ at 28 and $90 \mathrm{GPa}$, respectively [19], which are consistent with theoretical predictions [20,21]. Together with metallic hydrogen, these results suggest that superconductivity might be a ubiquitous phenomenon, accompanying molecular dissociation under pressure.

In this letter, we report an unexpected and unique structural evolution of $F_{2}$ molecular crystal under high pressure, obtained from extensive structure searches in conjunction with first principles calculations. Strikingly, instead of transforming directly from molecular phases to atomic or extended phases like $\mathrm{H}, \mathrm{N}$, and $\mathrm{O}$, Fluorine undergoes transitions into two intermediate structures, one that is stable from $2.8 \mathrm{TPa}$ to $4 \mathrm{TPa}$ consists of both $\mathrm{F}_{2}$ molecules and polymeric $\mathrm{F}$ chains, and the other that is stable from $4 \mathrm{TPa}$ to $30 \mathrm{TPa}$ consists of both $\mathrm{F}$ atoms and polymeric $\mathrm{F}$ chains. The true atomic phase of $\mathrm{F}$ can only be obtained under extremely high pressures beyond $30 \mathrm{TPa}$, clearly revealing the distinct resistance of $\mathrm{F}$ towards transition to an atomic phase. Despite the remaining molecular features in the intermediate structures, 
they are found to be superconducting, albeit with low $T_{\mathrm{c}}$ of only a few Kelvin.

The structure prediction is the key to computational materials discovery [22]. We searched for low enthalpy structures of fluorine in the multi-TPa range using the $a b$ initio random structure searching (AIRSS) method [23,24]. This approach has been applied to many systems including hydrogen [2,3], oxygen [10] and nitrogen [8] at high pressures. AIRSS based structure predictions were performed at selected pressures of $0.5,1,2,3,4,5,10,20,30,40 \mathrm{TPa}$ and different cell sizes containing up to 30 atoms. The validity of the potentials and the exchange correlation functionals have also been tested, the results are shown in Figs. S1-S3 of Supplemental Material [25]. Details of these computational methods and parameters are provided in the Supplemental Material.
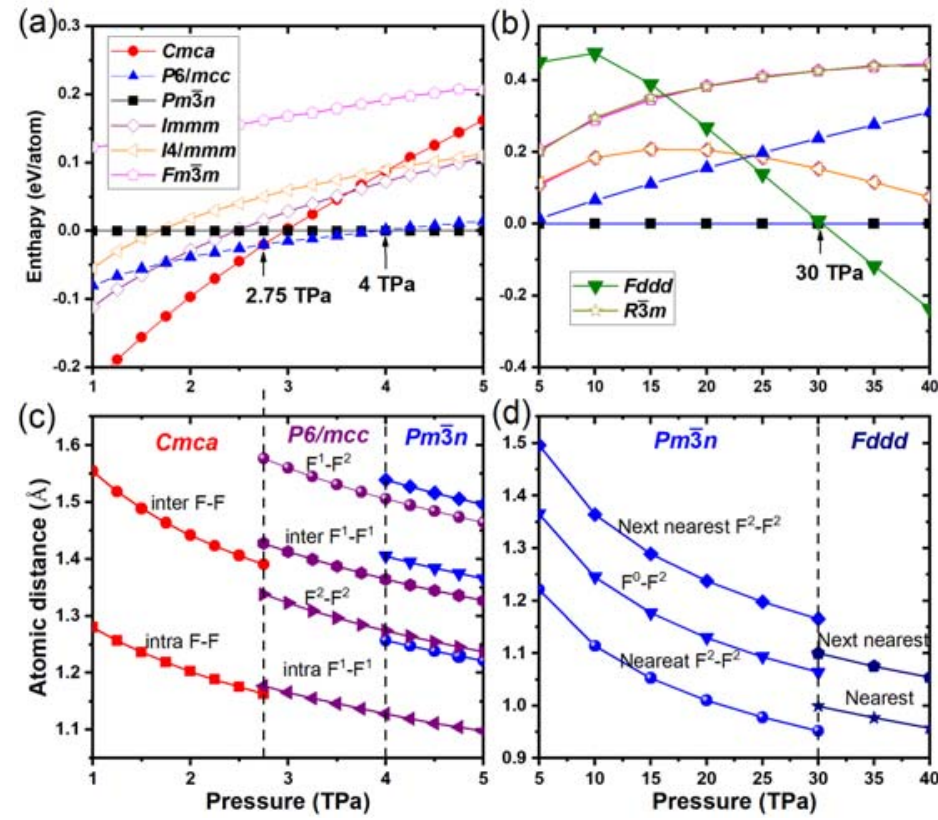

FIG. 1. (color online). (a) and (b) Enthalpies of various structures relative to the $\operatorname{Pm} \overline{3} n$ structure as functions of pressure. (c) and (d) The interatomic distances as function of pressure, including the nearest F-F distances (intra F-F) and the next nearest $\mathrm{F}-\mathrm{F}$ distances (inter $\mathrm{F}-\mathrm{F}$ ) in the $\mathrm{Cmca}$ phase; the nearest $\mathrm{F}^{1}-\mathrm{F}^{1}$ distance (intra $\mathrm{F}^{1}-\mathrm{F}^{1}$ ), the next nearest $\mathrm{F}^{1}-\mathrm{F}^{1}$ distance (inter $\mathrm{F}^{1}-\mathrm{F}^{1}$ ), the nearest $\mathrm{F}^{2}-\mathrm{F}^{2}$ distance, and the neighboring $\mathrm{F}^{1}-\mathrm{F}^{2}$ distance in the $P 6 / m c c$ phase; the nearest $\mathrm{F}^{2}-\mathrm{F}^{2}$ distance, the 
neighboring $\mathrm{F}^{0}-\mathrm{F}^{2}$ distance, the next nearest $\mathrm{F}^{2}-\mathrm{F}^{2}$ distance in the $P m \overline{3} n$ phase, the nearest and next nearest F- F distance in the Fddd phase.

Figure 1 shows the pressure dependence of enthalpy for structures that are found in this work as well as the most stable structures from previous studies. Strikingly, the molecular Cmca phase that was found to be stable from $8 \mathrm{GPa}$ to $100 \mathrm{GPa}$ persists up to $2.75 \mathrm{TPa}$. The low-pressure molecular phases such as $C 2 / m$ and $C 2 / c$ are omitted because they are only stable below $8 \mathrm{GPa}$ and their enthalpies are very close to that of Cmca. At $2.75 \mathrm{TPa}, \mathrm{F}$ transforms from the Cmca structure to a P6/mcc structure and then, at $4 \mathrm{GPa}$, to a $P m \overline{3} n$ structure. This structure is surprisingly stable in a large pressure range and only transforms to an $F d d d$ structure at $30 \mathrm{TPa}$. As shown by the calculated phonon spectra (Fig. S4), no structure shows dynamic instability in the pressure ranges that they are predicted to be thermodynamically stable.

Apart from the exceedingly wide range of stable pressures, the structures of these phases show a striking feature, namely the coexistence of atomic, molecular, and polymeric $\mathrm{F}$ atoms in the same structure. Although similar abnormal structural features were reported in amorphous $\mathrm{N}_{2}$ [32] and $\mathrm{SO}_{2}$ [33] recently, it remains a surprise that all the three different stages of structural evolution of the light p-block elements can coexist in thermodynamically stable crystalline phases. Among four stable structures, Cmca is a pure molecular phase and Fddd is a true atomic phase. The nearest F-F distances in Cmca at $1 \mathrm{TPa}$ are calculated to be $1.28 \AA$, while the next nearest F-F distances are $1.55 \AA$, which clearly shows the $F_{2}$ molecular character in this structure. For comparison, the bond length of $F_{2}$ molecules in gas phase is $1.43 \AA$. With increasing pressure, both nearest and next nearest F-F distances decrease but the molecular features remain throughout its stable pressure range.

In contrast to the Cmca and Fddd structures, there are two types of $\mathrm{F}$ atoms in the two intermediate structures, $\mathrm{F}^{1}$ and $\mathrm{F}^{2}$ in $P 6 / m c c$ and $\mathrm{F}^{2}$ and $\mathrm{F}^{0}$ in $P m \overline{3} n$. $\mathrm{TheF}^{1} / \mathrm{F}^{2}$ and $\mathrm{F}^{2} / \mathrm{F}^{0}$ ratios are 6:1 and 3:1 in these two structures, respectively. As shown in Fig. 2, P6/mcc consists of layers of $\mathrm{F}_{2}$ molecules formed by $\mathrm{F}^{1}$ atoms; 
whereas $\mathrm{F}^{2}$ atoms form straight polymeric chains that are perpendicular to the $F_{2}$ molecule planes. At $3 \mathrm{TPa}$, the nearest $\mathrm{F}^{1}-\mathrm{F}^{1}$ distance is $1.17 \AA$, and the next nearest $\mathrm{F}^{1}-\mathrm{F}^{1}$ distance is $1.41 \AA$ and is inside the $\mathrm{F}_{2}$ planes. In contrast, the nearest $\mathrm{F}^{2}-\mathrm{F}^{2}$ distance in the chain is $1.32 \AA$, indicating a weaker bond comparing with $\mathrm{F}^{1}-\mathrm{F}^{1}$. The neighboring $\mathrm{F}^{1}-\mathrm{F}^{2}$ distance is $1.56 \AA$, significantly larger than the neighboring distances between $\mathrm{F}$ atoms of the same type. Interestingly, the structural motifs of $\mathrm{F}_{2}$ planes are very similar to several high pressure structures of $\mathrm{H}_{2}$ [2], such as the $P 6_{3} / \mathrm{m}$ and $C 2 / c$ structures. In particular, the topology of the $\mathrm{F}_{2}$ planes in $P 6 / m c c$ structure is identical to that of the $\mathrm{H}_{2}$ planes in $P 6_{3} / m$ structure (see Fig.2 (b)). The major difference is that the vertical $\mathrm{H}$ atoms form $\mathrm{H}_{2}$ molecules in $P 6_{3} / m$, whereas the vertical $\mathrm{F}^{2}$ atoms in $P 6 / m c c$ form linear chains, as shown in Fig. 2 (a).

(a) $P 6 / m c c$

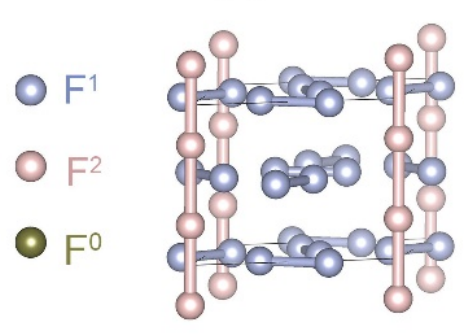

(c) $P m-3 n$ (b) $P 6 / m c c$

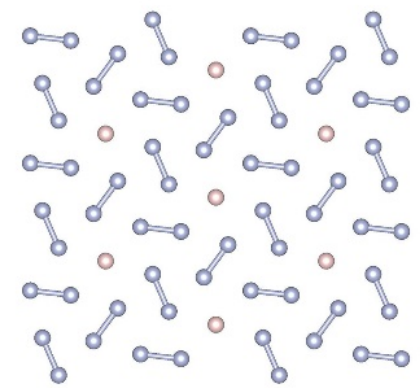

(e) Fddd

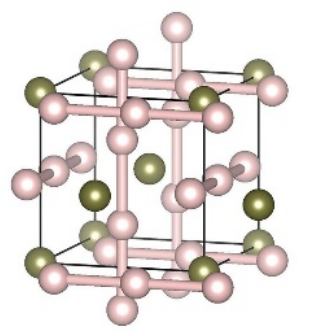

(d) $P m-3 n$

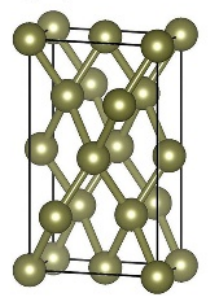

FIG. 2. (color online). (a) The crystal structure and (b) a layer of $P 6 / m c c$. The $\mathrm{F}^{1}, \mathrm{~F}^{2}$ and $\mathrm{F}^{0}$ are shown using blue, pink and dark yellow spheres, respectively. The layers are stacked in an ABCBA fashion. The crystal structure of $P m \overline{3} n$ showing (c) polymeric line chains and (d) icosahedron. (e) The crystal structure of atomic phase Fddd.

Although both the $P 6 / m c c$ and $P m \overline{3} n$ structures consist of two types of $\mathrm{F}$ atoms, 
the structural features of the two are distinctly different. Interestingly, $P m \overline{3} n$ is identical to A15 structures of some binary compounds such as $\mathrm{Cr}_{3} \mathrm{Si}$, except $\mathrm{F}$ plays the roles of both constituents in A15. The major difference to the $P 6 / m m c$ structure is that there are no $F_{2}$ molecules in this structure. This does not mean that $F$ has transformed into an atomic phase, because, as in all A15 structures, the $\mathrm{F}^{2}$ atoms form polymeric chains, with different bonding features to the $\mathrm{F}^{0}$ atoms. Correspondingly, at $5 \mathrm{TPa}$, the nearest $\mathrm{F}^{2}-\mathrm{F}^{2}$ distance in $P m \overline{3} n$ structure is $1.22 \AA$, which is slightly smaller than the $\mathrm{F}^{2}-\mathrm{F}^{2}$ distance of $1.24 \AA$ in P6/mcc line chains. Each $\mathrm{F}^{0}$ atom has 12 neighboring $\mathrm{F}^{2}$ atoms, with $\mathrm{F}^{0}-\mathrm{F}^{2}$ distance of $1.37 \AA$ at $5 \mathrm{TPa}$. The $\mathrm{F}^{2}$ atoms surrounding the $\mathrm{F}^{0}$ atom can be viewed as forming a deformed icosahedron. The distance of one edge of the distorted icosahedron is $1.22 \AA$ (the nearest $F^{2}-F^{2}$ ), and the other edge distance is $1.50 \AA$ (the next nearest $F^{2}-F^{2}$ ). Such cage structures are common in compounds formed by group 14 elements $(\mathrm{C}, \mathrm{Si}, \mathrm{Ge})$ at ambient condition, but rarely seen in solids under very high pressures. A well know exception is the $\mathrm{N}_{10}$ cage structure in diamondoid nitrogen [7].

(a) Cmca (100)

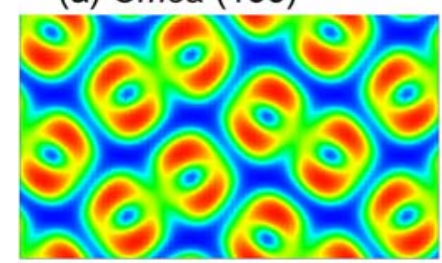

(c) $P 6 / m c c(100)$

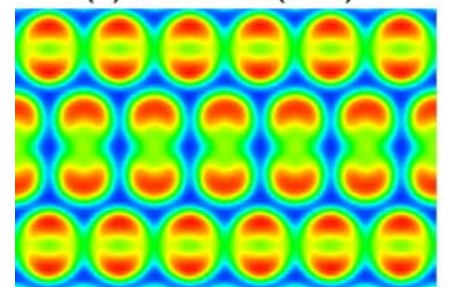

(b) $P 6 / m c c(001)$

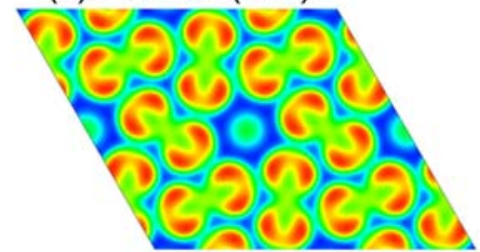

(d) $P m \overline{3} n(100)$

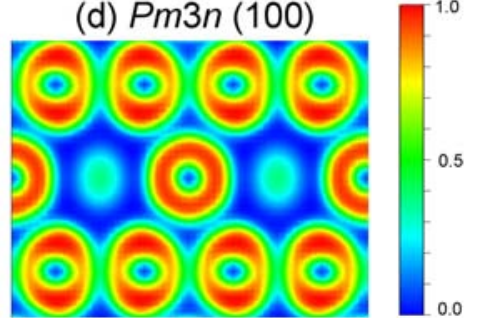

FIG. 3. The calculated ELF of our predicted phases. (a) Cmca in the (100) section at 3

TPa. (b) and (c) $P 6 / m c c$ in the (001) and (100) section at $3 \mathrm{TPa}$. (d) $P m \overline{3} n$ in the (100 section) at $5 \mathrm{TPa}$.

The mixed molecular and polymeric $\mathrm{F}$ in $P 6 / m c c$ and mixed polymeric and 
atomic $\mathrm{F}$ in $P m \overline{3} n$ structures are also revealed distinctively by their bonding nature. Electron Localization Functions (ELF) can distinguish the bond type and strength and is therefore calculated for the two structures. As shown in Fig. 3, ELF shows not only strong covalent bonds in $\mathrm{F}_{2}$ molecules in $\mathrm{Cmca}$ and $\mathrm{P6} / \mathrm{mcc}$ structures but also considerably stronger bonds between neighboring $\mathrm{F}^{2}$ atoms in the line chains in $P 6 / m c c$ and $P m \overline{3} n$ structures. The $\mathrm{F}^{1}$ atoms in neighboring molecules in the same $\mathrm{F}_{2}$ plane are also moderately bonded in $P 6 / m c c$ structure. On the other hand, ELF values between the $\mathrm{F}^{1}$ and $\mathrm{F}^{2}$ atoms in $P 6 / m c c$ and the $\mathrm{F}^{0}$ and $\mathrm{F}^{2}$ atoms in $P m \overline{3} n$ are small, indicating weak bonding between them.

The structural evolution of solid $\mathrm{F}$ under pressure is accompanied by changes in electronic structure. The molecular Cmca phase has a significant electronic gap, that decreases with pressure up to $500 \mathrm{GPa}$ and then increases with pressure throughout its stable pressure range. At $2.75 \mathrm{TPa}$, the transition pressure to $P 6 / m c c$ phase, its gap becomes $2.65 \mathrm{eV}$ as calculated by DFT using the PBE functional. The change of the band gap in $\mathrm{Cmca}$ structure is the result of the two competing effects. Under increasing pressure, it is reduced by the increasing intermolecular interactions, and increased by the contraction of the F-F bonds. In contrast to the molecular phase, the two intermediate phases, $P 6 / m c c$ and $P m \overline{3} n$, and the atomic phase, $F d d d$, are all metallic. Interestingly, as shown by the projected density of states (PDOS), the $\mathrm{F}_{2}$ molecules in $P 6 / m c c$ contribute significantly to the electron density at the Fermi level, demonstrating that the metallization might happen without regard to the dissociation of $\mathrm{F}_{2}$ molecules, similar to $\mathrm{H}_{2}$ and other halogens. Also, at the Fermi level of the P6/mcc structure, the PDOS of polymeric F $\left(\mathrm{F}^{2}\right)$ is very low and the PDOS of the molecular $\mathrm{F}\left(\mathrm{F}^{1}\right)$ show a dip, both leading to poor metallicity. In contrast, $P m \overline{3} n$ shows a much higher DOS at the Fermi level, with contributions from both the polymeric $\mathrm{F}\left(\mathrm{F}^{2}\right)$ and the atomic $\mathrm{F}\left(\mathrm{F}^{0}\right)$. Furthermore, $F d d d$, as an atomic phase, shows a typical band structure of metals and a high DOS at the Fermi level. We also 
calculated the band structure and the density of sates of $P 6 / m c c$ using the Hyed-Scuseria-Ernzerhof (HSE06) hybrid functional [34]. Although the results show slight changes, for example, the HSE06 functional opens a small gap at the $\Gamma$ point; the major features of the electronic structure remain the same. (Fig. S5).
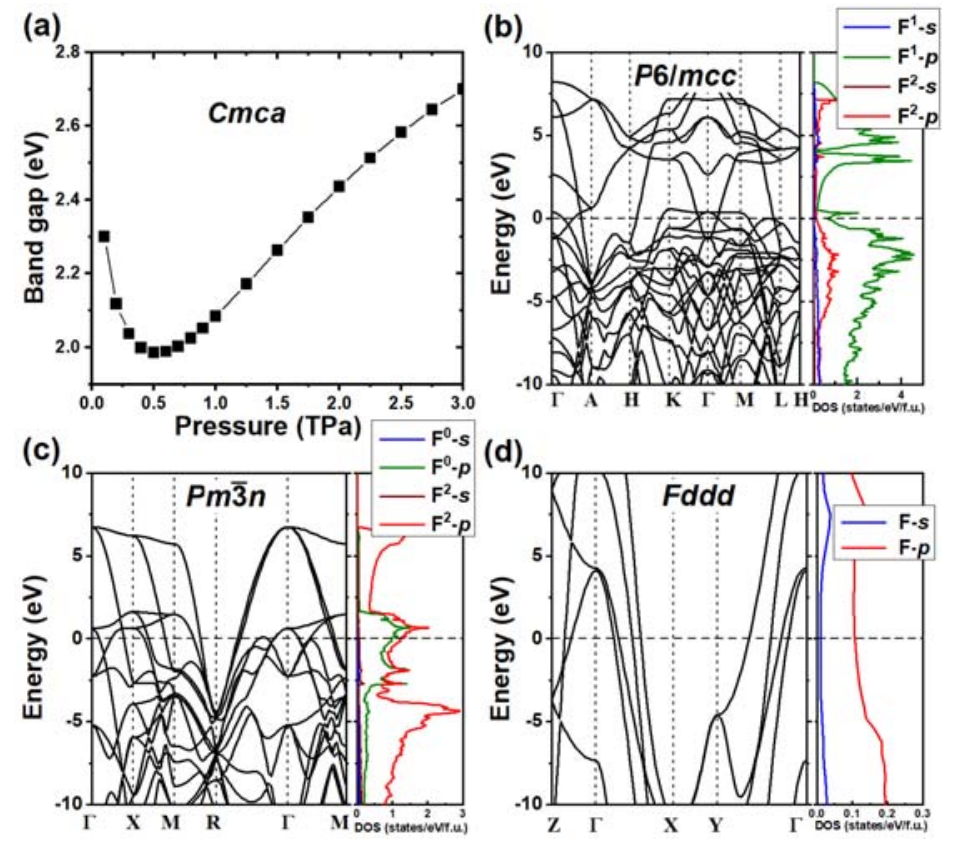

FIG. 4. (color online) (a) Variation of band gaps with pressure for Cmca. Band structure and partial densities of states (PDOS) of (b) P6/mcc phase at $3 \mathrm{TPa}$, (c) $P m \overline{3} n$ Phase at $5 \mathrm{TPa},(\mathrm{d}) F d d d$ at $30 \mathrm{TPa}$.

Since the intermediate $P 6 / m c c$ and $P m \overline{3} n$ structures, and the atomic $F d d d$ structure are metallic, they are attractive candidates for superconductivity, especially considering that both $\mathrm{I}$ and $\mathrm{Br}$ become superconducting in their molecular phase. Although the $T_{\mathrm{c}}$ of $\mathrm{I}$ and $\mathrm{Br}$ are below $2 \mathrm{~K}, \mathrm{~F}$ solids might exhibit higher phonon frequency and stronger electron-phonon coupling because of its lower atomic mass and stronger covalent bonds, both factors might improve $T_{\mathrm{c}}$. The Eliashberg function, electron-phonon coupling (EPC) constant and logarithmic average phonon frequency $\omega_{\log }$ are calculated to investigate the superconductivity (Table SI in supplementary information). For $P 6 / m c c$ structure, the EPC calculation yields a small $\lambda$ of 0.29 and a large $\omega_{\log }$ of $1382 \mathrm{~K}$ at $3 \mathrm{TPa}$. In the case of $P m \overline{3} n$ and $F d d d$ phases, the calculated $\lambda$ 
is 0.38 and 0.44 , and $\omega_{\log }$ is $1923 \mathrm{~K}$ and 2224 at $5 \mathrm{TPa}$ and $30 \mathrm{TPa}$, respectively. The $T_{\mathrm{c}}$ for three phases are calculated using the Allen-Dynes-modified McMillan equation [31]

$$
T_{C}=\frac{\omega_{\log }}{1.2} \exp \left[\frac{1.04(1+\lambda)}{\lambda-\mu^{*}(1+0.62 \lambda)}\right]
$$

where $\mu^{*}$ is the Coloumb pseudopotential, with typical values 0.10 and 0.13 . The calculated $T_{\mathrm{c}}$ is low at $0.3-1.3 \mathrm{~K}$ for the $P 6 / m c c$ structure at $3 \mathrm{TPa}, 2.1-5.5 \mathrm{~K}$ for the $P m \overline{3} n$ structure at $5 \mathrm{TPa}$, and $8.4-16.1 \mathrm{~K}$ for $F d d d$ at $30 \mathrm{TPa}$. The predicted $T_{\mathrm{c}}$ for atomic phase is larger than that of two intermediate phases, which due to the large $\lambda$ and $\omega_{\log }$.
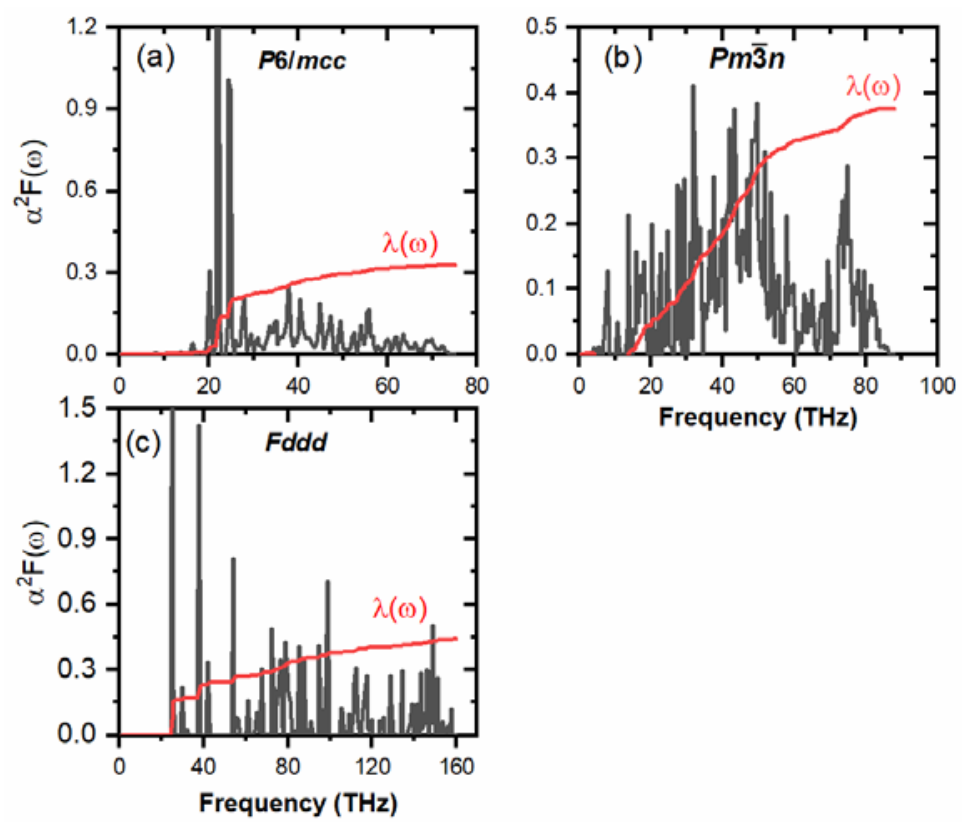

FIG. 5. (color online) The calculated Eliashberg function $\alpha^{2} \mathrm{~F}(\omega)$ (black) and EPC coefficient $\lambda$ (red) of (a) $P 6 / m c c$ at $3 \mathrm{TPa}$, (b) $P m \overline{3} n$ at $5 \mathrm{TPa}$, and (c) $F d d d$ at $30 \mathrm{TPa}$.

Finally, we discuss the cause of this unconventional multistep structural evolution toward the complete dissociation of the F-F bonds and the transformation of solid F to the atomic phase. Firstly, the internal energy $U$ and the $p V$, the two terms of enthalpy, are shown separately as functions of pressure for the four stable structures (see Fig. S6). It reveals that the major driving force for the structure evolution under pressure is the volume reduction. The results reveal very different trend of $\mathrm{pV}$ and $\mathrm{U}$ under 
pressure. Under an increasing pressure up to $5 \mathrm{TPa}$, the intermediate structures show an improving advantage due to the reduced volume relative to Cmca structure. Their internal energies are higher than that of the Cmca structure, but the differences dwindle. It is the combination of $U$ and $\mathrm{pV}$ that stabilizes these intermediate phases. Between the two intermediate phases, $P m-3 n$ has smaller volume at both low and high pressures. Its internal energy is higher but becomes smaller than P6/mcc at $27 \mathrm{TPa}$. Comparing with these structures, the atomic phase Fddd shows a higher internal energy throughout the pressure range. However, its volume and therefore its $\mathrm{pV}$ reduces rapidly with increasing pressure. The $\mathrm{pV}$ term becomes lower than $P 6 / m c c$ at $6 \mathrm{TPa}$ and lower than Pm-3n at $8 \mathrm{TPa}$. However, only at $30 \mathrm{TPa}$, when the reduction in $\mathrm{pV}$ overcomes the large excess $\mathrm{U}$, does Fddd become the most stable structure. Therefore, the transformation of $\mathrm{F}$ to the atomic phase is mainly driven by the volume reduction under pressure. This is in marked difference to $\mathrm{H}$ of which the atomic phase is caused by both the volume reduction and the change of the electronic structure.

The intricate structural evolution of $\mathrm{F}$ and the general trend of $\mathrm{pV}$ and $\mathrm{U}$ under pressure is largely due to the competition between reduced volume and the lone pair electrons of $\mathrm{F}$ that, compared to all other elements, is the hardest to compress. Although the atomic phase has the smallest volume that favors its formation under pressure, the complete quench of the lone pairs in this structure greatly increases its internal energy. In the pressure range from 2.8 to $30 \mathrm{TPa}, \mathrm{F}$ solid compromises the volume reduction and the quench of the lone pairs by adopting the two intermediate structures. In these structures, the lone pairs are only partially quenched because the open interstitial spaces leave room for lone pairs although they are compelled to deform and re-hybridize in these structures. The reduced volume eventually overcomes the large internal energy increase of the atomic structure caused by the quench of the lone pairs, however, the required pressure is much higher than that for other elements, including $\mathrm{H}$ and $\mathrm{O}$.

In summary, using a crystal structure prediction method, based on DFT calculations, we have thoroughly studied the structural evolution of solid F under high 
pressure and found a unique multistep transformation from low pressure molecular structures to an atomic phase, which has not been seen in any other elementary solid. Comparing with $\mathrm{H}, \mathrm{N}, \mathrm{O}$ and other halogens, $\mathrm{F}$ is extremely resistant to becoming an atomic phase and only reaches that destination of all elements under an exceedingly high pressure of $30 \mathrm{TPa}$. Most strikingly, the structural evolution undergoes two intermediate steps with high-symmetry structures, $P 6 / m c c$ and $P m \overline{3} n$, that contain the mixture of $F_{2}$ molecules and $F$ line chains and the mixture of $F$ line chains and $F$ atoms, respectively. Both two intermediate structures and the eventual atomic phase with Fddd symmetry are metallic and become superconducting, although the $T_{\mathrm{c}}$ are only at the level of 1 to a few Kelvins.

\section{ACKNOWLEDGEMENTS}

This work was supported by the National Key R\&D Program of China (No. 2018YFA0305900), National Natural Science Foundation of China (Nos. 11674122, 51632002 and 52072188). CJP acknowledges financial support from the Engineering and Physical Sciences Research Council [Grant EP/P022596/1]. MSM acknowledges the support of NSF CAREER fund (1848141) and the ACS PRF fund (59249-UNI6). Parts of the calculations were performed in the High Performance Computing Center

(HPCC) of Jilin University and TianHe-1(A) at the National Supercomputer Center in Tianjin.

Corresponding author : *cuitian@,nbu.edu.cn/cuitian@jlu.edu.cn

†miaoms@gmail.com 


\section{REFERENCES}

[1] J. M. McMahon and D. M. Ceperley, Ground-State Structures of Atomic Metallic Hydrogen, Phys. Rev. Lett. 106, 165302 (2011).

[2] C. J. Pickard and R. J. Needs, Structure of phase III of solid hydrogen, Nature Phys. 3, 473 (2007).

[3] C. J. Pickard, M. Martinez-Canales, and R. J. Needs, Density functional theory study of phase IV of solid hydrogen, Phys. Rev. B 85, 214114 (2012).

[4] R. T. Howie, C. L. Guillaume, T. Scheler, A. F. Goncharov, and E. Gregoryanz, Mixed Molecular and Atomic Phase of Dense Hydrogen, Phys. Rev. Lett. 108, 125501 (2012).

[5] A. F. Goncharov, E. Gregoryanz, H.-k. Mao, Z. Liu, and R. J. Hemley, Optical Evidence for a Nonmolecular Phase of Nitrogen above 150 GPa, Phys. Rev. Lett. 85, 1262 (2000).

[6] M. I. Eremets, R. J. Hemley, H.-k. Mao, and E. Gregoryanz, Semiconducting non-molecular nitrogen up to $240 \mathrm{GPa}$ and its low-pressure stability, Nature 411, 170 (2001).

[7] X. Wang et al., Cagelike Diamondoid Nitrogen at High Pressures, Phys. Rev. Lett. 109, 175502 (2012).

[8] J. Sun, M. Martinez-Canales, D. D. Klug, C. J. Pickard, and R. J. Needs, Stable All-Nitrogen Metallic Salt at Terapascal Pressures, Phys. Rev. Lett. 111, 175502 (2013).

[9] L. Zhu, Z. Wang, Y. Wang, G. Zou, H.-k. Mao, and Y. Ma, Spiral chain $\mathrm{O}_{4}$ form of dense oxygen, Proc. Natl. Acad. Sci. U.S.A. 109, 751 (2012).

[10] J. Sun, M. Martinez-Canales, D. D. Klug, C. J. Pickard, and R. J. Needs, Persistence and Eventual Demise of Oxygen Molecules at Terapascal Pressures, Phys. Rev. Lett. 108, 045503 (2012).

[11] T. H. Jordan, W. E. Streib, and W. N. Lipscomb, Single Crystal X Ray Diffraction Study of B Fluorine, J. Chem. Phys. 41, 760 (1964).

[12] Q. Lv, X. Jin, T. Cui, Q. Zhuang, Y. Li, Y. Wang, K. Bao, and X. Meng, Crystal structures and electronic properties of solid fluorine under high pressure, Chin. Phys. B 26, 076103 (2017).

[13] Q. Zeng, Z. He, X. San, Y. Ma, F. Tian, T. Cui, B. Liu, G. Zou, and H. k. Mao, A new phase of solid iodine with different molecular covalent bonds, Proc. Natl. Acad. Sci. U.S.A. 105, 4999 (2008).

[14] T. Kenichi, S. Kyoko, F. Hiroshi, and O. Mitsuko, Modulated structure of solid iodine during its molecular dissociation under high pressure, Nature 423, 971 (2003).

[15] Y. Fujii, K. Hase, N. Hamaya, Y. Ohishi, A. Onodera, O. Shimomura, and K. Takemura, Pressure-induced face-centered-cubic phase of monatomic metallic iodine, Phys. Rev. Lett. 58, 796 (1987).

[16] D. Duan, Y. Liu, Y. Ma, Z. Liu, T. Cui, B. Liu, and G. Zou, Ab initio studies of solid bromine under high pressure, Phys. Rev. B 76, 104113 (2007).

[17] P. Li, G. Gao, and Y. Ma, Modulated structure and molecular dissociation of solid chlorine at high pressures, J. Chem. Phys. 137, 064502 (2012).

[18] P. Dalladay-Simpson, J. Binns, M. Peña-Alvarez, M.-E. Donnelly, E. Greenberg, V. Prakapenka, X.-J. Chen, E. Gregoryanz, and R. T. Howie, Band gap closure, incommensurability and molecular dissociation of dense chlorine, Nat. Commun. 10, 1134 (2019).

[19] K. Shimizu, K. Amaya, and N. Suzuki, Pressure-induced Superconductivity in Elemental Materials, J. Phys. Soc. Jpn. 74, 1345 (2005).

[20] D. Duan, X. Jin, Y. Ma, T. Cui, B. Liu, and G. Zou, Effect of nonhydrostatic pressure on 
superconductivity of monatomic iodine: An ab initio study, Phys. Rev. B 79, 064518 (2009).

[21]D. Duan et al., The crystal structure and superconducting properties of monatomic bromine, J. Phys.: Condens. Matter 22, 015702 (2010).

[22] A. R. Oganov, C. J. Pickard, Q. Zhu, and R. J. Needs, Structure prediction drives materials discovery, Nat. Rev. Mater. 4, 331 (2019).

[23] C. J. Pickard and R. J. Needs, High-pressure phases of silane, Phys. Rev. Lett. 97, 045504 (2006).

[24] C. J. Pickard and R. J. Needs, Ab initiorandom structure searching, J. Phys.: Condens. Matter 23, 053201 (2011).

[25] See Supplemental Material for computational details, the functionals and pseudopotentials test, phonon dispersion curves, $\mathrm{pV}$ and internal energy $\mathrm{U}$ with pressure, the calculated $\mathrm{Tc}$ and structural information of all predicted structures, which includes Refs. [23, 24, 26-31].

[26] M. D. Segall, J. D. L. Philip, M. J. Probert, C. J. Pickard, P. J. Hasnip, S. J. Clark, and M. C. Payne, First-principles simulation: ideas, illustrations and the CASTEP code, J. Phys.: Condens. Matter 14, 2717 (2002).

[27]J. P. Perdew, K. Burke, and M. Ernzerhof, Generalized Gradient Approximation Made Simple, Phys. Rev. Lett. 77, 3865 (1996).

[28] G. Kresse, and J. Furthmüller, Efficient iterative schemes for ab initio total-energy calculations using a plane-wave basis set. Phys. Rev. B 54, 11169 (1996).

[29] A. Togo, F. Oba, and I. Tanaka, First-principles calculations of the ferroelastic transition between rutile-type and $\mathrm{CaCl}_{2}$-type $\mathrm{SiO}_{2}$ at high pressures, Phys. Rev. B 78, 134106 (2008).

[30] G. Paolo et al., QUANTUM ESPRESSO: a modular and open-source software project for quantum simulations of materials, J. Phys.: Condens. Matter 21, 395502 (2009).

[31] P. Blaha, K. Schwarz, P. Sorantin, and S. Trickey, Full-potential, linearized augmented plane wave programs for crystalline systems, Comput. Phys. Commun. 59, 399 (1990).

[32] D. Melicherová, O. Kohulák, D. Plašienka, and R. Martoňák, Structural evolution of amorphous polymeric nitrogen from ab initio molecular dynamics simulations and evolutionary search, Phys. Rev. Mater. 2, 103601 (2018).

[33] H. Zhang et al., Pressure-induced amorphization and existence of molecular and polymeric amorphous forms in dense $\mathrm{SO}_{2}$, Proc. Natl. Acad. Sci. U.S.A. 117, 8736 (2020).

[34] A. V. Krukau, O. A. Vydrov, A. F. Izmaylov, and G. E. Scuseria, Influence of the exchange screening parameter on the performance of screened hybrid functionals, J. Chem. Phys. 125, 224106 (2006).

[35] P. B. Allen and R. C. Dynes, Transition temperature of strong-coupled superconductors reanalyzed, Phys. Rev. B 12, 905 (1975). 